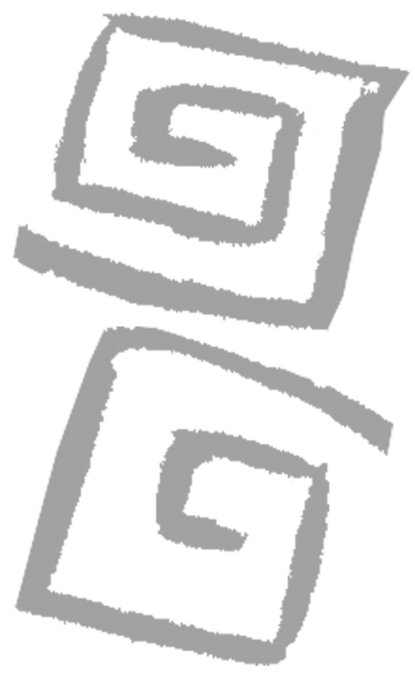

\title{
Información, autoayuda y creación de identidades: las tecnologías de la información y la comunicación (TIC) y las asociaciones de discapacitados físicos. El ejemplo de la poliomielitis
}

\author{
Information, self-help and identity creation: information \\ and communication technologies (ICTs) and associations \\ for the physically disabled. The example of poliomyelitis
}

Ballester, Rosa ${ }^{1}$; Bueno, Eduardo ${ }^{2}$; Sanz-Valero, Javier ${ }^{3}$

\footnotetext{
${ }^{1}$ Doctora en Medicina. Catedrática de Historia de la Ciencia, Universidad Miguel Hernández, España. Rosa.Ballester@umh.es

${ }^{2}$ Licenciado en Historia. Becario del Proyecto Prometeo para grupos de excelencia (Prometeo 2009/122), Generalitat Valenciana, España. ebueno@umh.es

${ }^{3}$ Doctor en Salud Pública. Profesor del Departamento de Salud Pública, Historia de la Ciencia y Ginecología, Universidad Miguel Hernández, España. Profesor del Departamento de Enfermería Comunitaria, Medicina Preventiva y Salud Pública e Historia de la Ciencia, Universidad de Alicante, España. jsanz@umh.es
}

RESUMEN El objetivo del trabajo es estudiar la calidad de las páginas web de asociaciones de personas afectadas por poliomielitis y síndrome postpolio, como uno de los sistemas de intercambio de información y de visibilidad social de las personas con discapacidades físicas. Se realizó un estudio descriptivo de carácter transversal; fueron evaluadas 375 páginas web que cumplían los criterios de inclusión, encontradas a través del motor de búsqueda Google. La calidad fue estudiada a través de 22 variables, 8 de las cuales conforman el "Indicador de Credibilidad". Ninguna de las páginas cumplió con todos los criterios de calidad ni con los 8 puntos del Indicador de Credibilidad. Se observa una correlación positiva entre las variables de calidad y los criterios de credibilidad ( $r$ de Pearson $=0,81, p<0,001$ ), por lo que se sugiere el Indicador de Credibilidad como una herramienta útil para determinar la calidad de las páginas web. Su uso debe complementarse con otro tipo de acercamientos cualitativos que exploren el contexto de las relaciones expertos-legos y las funciones que dichas páginas cumplen.

PALABRAS CLAVE Internet; Indicadores de Calidad; Grupos de Autoayuda; Poliomielitis; Síndrome Postpoliomielitis.

ABSTRACT The aim of this paper is to study the quality of the websites of associations for people with polio and post-polio syndrome as an example of an important method for exchanging information and promoting the social visibility of physically disabled people. A descriptive cross-sectional study of 375 sites that met inclusion criteria, found using of the Google search engine, was carried out. The quality was studied through 22 variables, 8 of which make up the "Credibility Indicator". None of the websites met all the quality criteria nor all 8 items in the Credibility Index. A positive correlation between the variables of quality and credibility criteria (Pearson $r=0.81$, $\mathrm{p}<0.001$ ) was observed, therefore the Credibility Index is suggested as a useful tool for determining the quality of the websites. However, this tool must be supplemented with other qualitative approaches that explore both the contexts of lay-expert relationships and the function these sites serve.

KEY WORDS Internet; Quality Indicators; Self-Help Groups; Poliomyelitis; Postpoliomyelitis Syndrome. 


\section{INTRODUCCIÓN}

En los últimos años se han multiplicado los estudios sobre el uso de diversas herramientas procedentes de las tecnologías de la información y la comunicación (TIC) en el mundo de las discapacidades. Una de las facetas contempladas ha sido, por un lado, desarrollar sistemas que faciliten la relación en grupos, el trabajo cooperativo y nuevas formas de interacción social entre estas personas, en una suerte de lo que se han venido en denominar sistemas de "espacio social aumentado" (1) y, en segundo término, analizar su uso efectivo en la práctica.

La relación de las $\mathrm{TIC}$ con el complejo universo de las discapacidades físicas y de las asociaciones relacionadas con ellas hay que enmarcarlo, al menos, en un doble eje de referencia. Por un lado, desde el punto de vista del fenómeno de asociacionismo de personas afectadas por algún importante problema de salud y de los mecanismos de comunicación de los grupos de autoayuda. En segundo término, encuadrando el análisis de las páginas web de estas asociaciones en el marco de los estudios sobre calidad de la información en salud y enfermedad existentes en Internet. En este último terreno, contamos ya con una sólida línea de trabajos que nos van a servir como modelo para nuestro propósito (2-4).

Las asociaciones de pacientes forman parte de un universo más amplio que se manifiesta en la proliferación de grupos de ayuda mutua, así como en la reivindicación creciente de los usuarios a favor de una participación más activa en la concepción de los servicios destinados a ellos (5). Su punto de partida contemporáneo hay que situarlo a mediados del siglo XX (6), como una de las modalidades que adopta la sociedad civil para agruparse con fines concretos (7). El hecho de que, en algunos casos como el de la poliomielitis, la mayoría de los afectados entre los años 1950 y 1960, fueran niños, hizo que los padres se implicaran negándose a arrojar la toalla ante una enfermedad para la que no había terapéuticas eficaces. Estas asociaciones se plantearon no solo como el lugar para la elaboración de una identidad colectiva y como apoyo de reintegración social de los enfermos y sus familias sino que, además, proyectaron la enfermedad al dominio público, organizando (como en el caso de la Asociación de Paralíticos de Francia) jornadas de estudios y reuniones de información dirigidas a un público especializado y también lego. Curiosamente, 30 o 40 años más tarde, a partir de la década del 80 del siglo pasado, algunos de aquellos niños que habían sobrevivido a la polio y habían conseguido sobreponerse a sus secuelas, fueron afectados por una nueva patología muy poco conocida por los médicos: el síndrome postpolio, que golpeó de nuevo sus vidas con otros problemas, muchas veces no reconocidos a los efectos de la reivindicación de situaciones de invalidez. Esta situación ha sido el acicate más importante que explica el elevado número de asociaciones que suelen denominarse de polio y síndrome postpolio, en muchos países occidentales.

Un tema muy interesante es el de las relaciones de los miembros de estas asociaciones con los expertos, clínicos e investigadores. Rabehasiora y Callon (5) identifican tres tipos de asociaciones de pacientes. La asociación auxiliar, en la que el médico decide el tratamiento más pertinente, da algún tipo de información al paciente y se espera que este coopere, sin más. En el segundo tipo, en lugar de remitirse pura y simplemente a los especialistas, la asociación de enfermos se dota de los medios para adquirir los conocimientos necesarios para discutir con ellos. Sería, sobre todo, fruto del activismo de ciertas asociaciones que, ante la gravedad de los problemas y la falta de seguridad de los expertos, deciden compartir informaciones y transformarse en legos-expertos. Finalmente, el tercer modelo es la asociación de partenariado. En el centro del compromiso de la asociación de enfermos prototípica de este perfil, el enfermo se considera indispensable para la toma de decisiones ya que es un "experto en experiencias", lo que significa que deben buscarse procedimientos para recoger sistemáticamente estas experiencias, integrarlas en el cuerpo de conocimientos y poner a punto las buenas prácticas (8).

La convicción de que el acceso de las personas discapacitadas a la sociedad de la información es un elemento clave para mejorar sus condiciones de vida, su integración, participación y desarrollo como ciudadanos de pleno 
derecho, es un hecho reconocido socialmente, al menos, a nivel programático. El desarrollo de herramientas cada vez más sofisticadas de comunicación y procesamiento de la información puede suponer, para ese importante segmento de la ciudadanía, añadir nuevas barreras, pero si esta tecnología es utilizada de forma adecuada, se transforma en una ayuda fundamental para que estas barreras puedan ser superadas y posibilita a las personas con discapacidades la accesibilidad a servicios de los que, de otra forma, se verían excluidos.

La iniciativa e-Europe, desarrollada por el Consejo de la Unión Europea desde 2003, coincidiendo con el Año Europeo de las Personas con Discapacidad, es un ejemplo paradigmático de este cambio de mentalidad al indicar que "debe prestarse una atención especial a las personas con discapacidad para evitar la info-exclusión, asegurando su total pertenencia a la Sociedad de la Información" (1). Las actualizaciones de estos presupuestos se han visto plasmadas en sucesivos informes y recomendaciones en aras de orientar la investigación en materia de TIC y discapacidad. Proyectos de investigación recientes han desarrollado interfaces multimodales y multimedia inteligentes, que dan una dimensión nueva a la comunicación hombre-máquina, utilizables por personas en situación de discapacidad. Otra línea de trabajo se orienta en el sentido de cómo debe enfocarse el soporte social en actividades de comunicación de y con estas personas (9).

Sin embargo, no se ha prestado el mismo interés al análisis intrínseco de los sistemas mediante los cuales se establecen, de hecho, redes sociales de apoyo y soporte a través de las tecnologías. Una modalidad, los Ilamados "computer-mediated support groups" han sido estudiados en asociaciones de pacientes con problemas oncológicos (10) y también en las discapacidades en general (11).

El objetivo de este trabajo es un estudio de campo, focalizado en las páginas web que aportan información de cualquier índole sobre la polio y de las asociaciones de afectados de polio y síndrome postpolio, como uno de los medios a través de los cuales las personas con este tipo de discapacidades físicas se expresan y comunican.

\section{MÉTODO}

Se realizó un estudio descriptivo de carácter transversal en una muestra de páginas web accesibles a través de las referencias obtenidas de la búsqueda en Google. Para que dicha muestra no se ciñera a un ámbito geográfico o lingüístico concreto, se optó por usar la versión internacional del buscador (http://www.google.com/), e introducir la palabra clave "polio", que comparten, por ejemplo el inglés y el español, mientras que se buscó "association" por ser mayoritario el inglés en los contenidos web.

\section{Recopilación de datos}

Los datos fueron obtenidos directamente a través de la búsqueda y acceso a las páginas web. Se realizaron dos búsquedas, por un lado introduciendo la palabra clave "polio" y, por otro, las palabras "polio" y "association". Para evitar cambios en los resultados y mantener el PageRank de Google, optamos por una "búsqueda avanzada" que mostrara 100 resultados por página y guardamos las referencias obtenidas en formato PDF con su hipervínculo correspondiente, de modo que pudiésemos acceder a las web con posterioridad. En el estudio tuvimos en cuenta la ecuación PageRank (los primeros 20 resultados tienen el $80 \%$ de probabilidades de ser consultados por los internautas) y a fin de neutralizarlo, se analizaron las 20 páginas web mejor posicionadas. Además, se tuvo presente la falacia muestral de Google: el total proporcionado de las web no es más que una estimación, ya que las búsquedas del motor Google no ofrecen más allá de 1.000 resultados. Ambos factores nos permitieron recoger una muestra representativa del objeto de estudio.

\section{Criterios de inclusión}

Para las búsquedas de "polio" fueron todas aquellas páginas web que arrojaran alguna información sobre la enfermedad, fuese como eje temático central, o se tratase de forma tangencial. En cuanto a la búsqueda de "polio" y "association", se incluyeron únicamente las páginas pertenecientes a grupos de ayuda de antiguos enfermos (survivors) y actuales afectados de síndrome postpolio. 


\section{Criterios de exclusión}

Aquellas páginas web que solicitaran un registro o pago adelantado por su acceso, dado que no suelen ser consultadas por la población en general. Para el caso concreto de las relacionadas con las palabras clave "polio" y "association" se excluyeron todas aquellas que no remitieran a una asociación o lugar de apoyo para afectados de polio o síndrome postpolio. Por ejemplo, se excluyeron las elaboradas por la Organización Mundial de la Salud y las que remitían a clínicas o profesionales como neurólogos o especialistas en patologías neuromusculares. También fueron excluidas para el análisis las páginas que carecían de contenidos y, simplemente, remitían a otras mediante enlaces.

\section{Muestra del estudio}

Para calcular la muestra se hizo una estimación de la población con tendencia a infinito (aproximadamente esperamos un valor de 0,5, intervalo de precisión de 0,05, y un intervalo de confianza $=0,95)$, teniendo en cuenta la falacia muestral de Google. Una vez realizada la búsqueda utilizando la palabra "polio", obtuvimos 399 hipervínculos: 386 a través de un muestreo aleatorio simple sin reemplazo, a los que sumamos 13 de los 20 resultados correspondientes a las dos primeras páginas propuestas por el buscador, dado que 7 de estas páginas ya figuraban en el muestreo aleatorio simple. Para la búsqueda y el examen de las páginas web con los términos "polio" y "association" (que nos remitió a las asociaciones de afectados de polio y síndrome postpolio, mencionadas más arriba), obtuvimos idénticos números. En suma, se obtuvo una muestra de 798 páginas web (399 para el término "polio" y 399 para los términos "polio" y "association"), de las que se analizaron 375 páginas (320 para el primer caso, 55 para el segundo) atendiendo a los criterios de inclusión y exclusión expuestos.

\section{Tratamiento de la información}

El almacenamiento de los datos se realizó mediante la hoja de cálculo Excel $2010^{\circledR}$ que facilitó un acceso permanente a las páginas web, la ejecución de cálculos matemáticos y la construcción de tablas. Los cálculos estadísticos se realizaron con el programa SPSS $^{\circledR}$, versión 15.0 para Windows. Se rellenaron dobles tablas para cotejar la igualdad de los resultados y evitar errores de transcripción.

\section{Análisis de datos}

Las variables cuantitativas son descritas con la media y la desviación estándar, las cualitativas son descritas con su valor absoluto y porcentaje. Se empleó la media y la mediana como medida de tendencia central. Para probar el significado de la diferencia entre las medias para muestras independientes fue empleada la prueba t de Student. La relación lineal entre dos variables cualitativas se verificó mediante el coeficiente de correlación de Pearson. El nivel de significación utilizado en las mediciones de pruebas de hipótesis fue $\alpha \leq 0,05$.

\section{Variables de estudio}

Se estudiaron detalladamente las siguientes variables y criterios: las recomendaciones propuestas en el Health Information Locator por la Organización Panamericana de la Salud (12), las ofrecidas por Dublin Core (13), la Health On the Net Foundation (14), la Web Médica Acreditada (15) y Netscoring (16). Las variables redundantes se eliminaron, quedando un total de 22 que fueron identificadas del siguiente modo: Autoría*, Filiación*, Equipo Editorial*, Fecha de Creación*, Fecha de Actualización*, Aval*, Acreditación*, Financiación*, Coherencia en el Título, Contacto, Validez de los tres primeros enlaces, Coherencia de los Enlaces, Ayudas, Gestión de la Información, Tamaño de la Letra, Declaración de Conflicto de Intereses, Objetividad, Estadísticas de Consulta, Buscador, Accesibilidad, Interoperabilidad y Política Editorial. Las primeras 8 variables marcadas con un asterisco componen el Indicador de Credibilidad.

A fin de evaluar la adecuación de todas las variables del estudio se usó la dicotomía "sí o no", de forma independiente, por dos investigadores. En caso de observar diferencias en los resultados, se consultó a un tercer observador llegándose a un consenso por mayoría. 


\section{RESULTADOS}

Conforme al cálculo de la muestra y atendiendo a las dos búsquedas mencionadas, encontramos:

- De los 399 resultados sobre "polio", se encontraron 17 enlaces rotos (IC95\% 2,28-6,24). De las restantes se dejaron de analizar 62 páginas web, 19 remitían a videos (IC95\% 2,67-6,85) y 6 a imágenes (IC95\% 0,31-2,7) sujetas a otra metodología de análisis, mientras que 37 (IC95\% 6,43-12,12) no versaban sobre la enfermedad estudiada. En total, dimos con 320 homepages (IC95\% 76,29-84,11) susceptibles de ser analizadas.

- De las 399 páginas web que recogen las palabras "polio" y "association", se hallaron 12 enlaces rotos (IC95\% 1,33-4,68). Atendiendo a los criterios de exclusión, fueron descartadas 332 páginas web (IC95\% 79,54-86,88), quedando finalmente un total de 55 (IC95\% 10,40$17,17)$ para su estudio.

\section{Cumplimiento de las variables relacionadas con la calidad}

El análisis descriptivo del cumplimiento de las 22 variables de calidad arrojó los siguientes datos: para las páginas web sobre "polio", los resultados fueron una media de 9,27 \pm 0,15 (IC95\% 8,97-9,56), mínimo de 1, máximo de 16 y mediana de 9; para las páginas web sobre "association", los resultados fueron una media de 7,76 $\pm 0,32$ (IC95\% 7,12-8,41), mínimo de 2, máximo de 13 y mediana de 8 . Observamos que ninguna página web encontrada cumplió todos los criterios de calidad. Además, pudimos apreciar diferencias significativas a favor de las páginas de polio, cuando se compararon los valores medios de los resultados obtenidos en el cumplimiento de las variables de calidad (prueba t para muestras independientes igual a 3,94, con 373 grados de libertad y $\mathrm{p}<0,001)$.

No podemos dejar de mencionar que buena parte de las páginas de contenidos sobre polio, en concreto, 97 (IC95\% 20,10-28,52), se correspondían con reseñas aparecidas en distintos lugares de información general (por ejemplo, versiones digitales de periódicos como el New York Times o portales de cadenas de televisión como la BBC). Tomando como muestra diferenciada aquellas web que podríamos agrupar bajo esta categoría, los resultados son los siguientes: media de 9,91 \pm 0,16 (IC95\% 9,59-10,23), máximo de 14, mínimo de 6, mediana de 10 . La calidad de este grupo, contrastada con el resto de páginas, arroja una diferencia significativamente mayor (prueba $t$ para muestras independientes igual a $-2,89$, con 318 grados de libertad $y$ $\mathrm{p}<0,004)$

\section{Cumplimiento de las variables pertenecientes al Indicador de Credibilidad}

Considerando el cumplimiento de las 8 variables que conforman este indicador, los resultados son los siguientes:

- Para las páginas web que contienen la palabra "polio", los resultados fueron una media de 3,18 \pm 0,08 (IC95\% 3,02-3,34), un mínimo de 0 , un máximo de 7 y una mediana de 3 variables.

- Para las páginas web que recogen los términos "polio" y "association", los resultados fueron una media de 2,25 \pm 0,15 (IC95\% 1,95-2,56), un mínimo de 0 , un máximo de 5 y una mediana de 2 variables.

Al igual que en el apartado anterior, se constataron diferencias significativas en lo que se refiere al cumplimiento de las variables de credibilidad (prueba t para muestras independientes igual a 4,42, con 373 grados de libertad $y$ $p<0,001)$

\section{Relación entre el cumplimiento de los criterios de calidad y el Indicador de Credibilidad}

Para las 375 páginas web estudiadas en conjunto (320 pertenecientes a la búsqueda con el término "polio", y 55 con "polio" y "association"), se observó la existencia de una correlación positiva entre el cumplimiento de los criterios de calidad y el Indicador de Credibilidad obteniendo una $r$ de Pearson $=0,81, p<0,001$.

Ocurre lo mismo cuando los ítems son estudiados de forma separada: 
- Valores para las páginas web con la palabra "polio": la $r$ de Pearson $=0,80, p<0,001$.

- Valores para las páginas web que contienen las palabras "polio" y "association": la r de Pearson $=0,74, p<0,001$.

A la vista de los datos extraídos, podemos señalar que la calidad total aumenta en proporción al crecimiento del Indicador de Credibilidad, concluyendo que a mayor presencia de este se produce un aumento de la calidad.

\section{Autoría y filiación como indicadores de una mayor calidad en las web}

De todas las páginas web estudiadas, 145 (IC95\% 33,37-43,15) indican tanto su autoría como su filiación, mostrando diferencias significativas en el cumplimiento de las variables de calidad respecto a aquellas otras que no lo hacen (prueba $t$ para muestras independientes igual a $-8,66$, con 373 grados de libertad y $p<0,001)$. Conclusiones similares obtenemos al relacionar la autoría y la filiación con el Indicador de Credibilidad (prueba t para muestras independientes igual a $-10,85$, con 373 grados de libertad y $p<0,001$ ).

Al repasar los dos grupos temáticos por separado, obtuvimos los siguientes resultados:

- Para las páginas web que contenían la palabra "polio", 130 (IC95\% 35,24-46,01) cumplían los criterios de autoría y filiación. Reconociendo diferencias significativas respecto a las que no lo cumplen en cuanto a la calidad (prueba t para muestras independientes igual a $-7,59$, con 318 grados de libertad y $p<0,001)$ e Indicador de Credibilidad (prueba t para muestras independientes igual a -9,52, con 318 grados de libertad y $\mathrm{p}<0,001)$

- Para las páginas web que contenían las palabras "polio" y "association", 15 (IC95\% 14,3136,53) observaban los criterios de autoría y filiación. En este caso, volvemos a constatar la significativa correspondencia entre las homepages que los cumplían y las que no en relación a la calidad (prueba t para muestras independientes igual a $-3,75$, con 53 grados de libertad y $p<0,001)$ y el Indicador de Credibilidad (prueba t para muestras indepen- dientes igual a -5,57, con 53 grados de libertad y $\mathrm{p}<0,001)$.

Resulta claro que las web que explicitan la persona responsable del contenido y la institución que la ampara, proporcionan mejores resultados generales que aquellas que no lo hacen.

\section{DISCUSIÓN}

Los resultados obtenidos en el análisis de las páginas web estudiadas, muestran un escaso cumplimiento de los criterios de calidad utilizando las herramientas arriba indicadas. Más claro aun resulta al constatar que los valores medios de cumplimiento en ningún caso han alcanzado la mitad de las 22 variables investigadas (11). La misma conclusión puede aplicarse a los datos obtenidos en el estudio respecto a las variables del Indicador de Credibilidad.

Por otro lado, los resultados alcanzados coinciden en gran medida con los obtenidos en estudios realizados en otras páginas web relativas a temas de salud que constituyen, como es bien sabido, un porcentaje alto de todas las que circulan por la red. Si nos situamos en el enfoque de las páginas web de asociaciones de afectados de polio como ejemplos de páginas de información en temas de salud/enfermedad en general, se plantean las cuestiones de cómo garantizar y valorar la calidad de sus contenidos y sobre el importante problema de la información obtenida en Internet, una información que se encuentra al alcance de usuarios no expertos, que no pueden, por ello, detectar un tipo de información falsa o no verificada. Hay una importante evidencia empírica de que los usuarios no tienden a evaluar la calidad de la información sobre salud que obtienen en la red o emplean una serie de criterios subjetivos para valorar la veracidad de la misma o la actualidad de esta. Algunas iniciativas como el estudio e-Europe 2002 insistía en la necesidad de intercambiar experiencias e información sobre cómo aplicar los criterios de calidad para ayudar a los usuarios que acceden a la información contenida en las páginas web relacionadas con la salud, pero todavía es un tema no resuelto. 
Pese a las dificultades que implica valorar adecuadamente la calidad de la web, la utilización de las 8 variables de credibilidad que hemos utilizado en nuestro trabajo, configuran un protocolo simple y útil para poder evaluar una web de forma rápida y fácil, permitiendo manejar los recursos de Internet con un mínimo de garantía y veracidad y se han mostrado de utilidad. Uno de los elementos más significativos para la evaluación ha sido el relativo a los autores ligados a una institución, uno de los primeros criterios de calidad que deberían tomarse en consideración. Internet recoge una gran cantidad de opiniones individuales que deben ser convenientemente pasadas por el tamiz crítico de la vinculación de esta o estas personas a un organismo que garantice su rigor científico.

En cuanto a otro tipo de resultados, se observa que el número de enlaces inactivos o rotos -la accesibilidad de las páginas web- era ligeramente más bajo que el que se manejaba en otro tipo de temas utilizando este mismo instrumento de evaluación $(4,17,18)$.

Las limitaciones del estudio son fundamentalmente de tres tipos. Por un lado, que los términos utilizados en la indización (polio, association) son muy específicos y por ello no se recupera información de otro tipo de páginas de asociaciones que figuran bajo denominaciones más amplias como, por ejemplo, los distintos Foros de Vida Independiente (que incluyen, entre sus asociados, a personas con polio) o las asociaciones de discapacidades físicas en general.

En segundo lugar, hay que tomar en consideración que la información científica allí incluida, relativa a la enfermedad poliomielítica, cuyo rigor es el evaluado a través de los instrumentos utilizados en este trabajo (especialmente en lo relativo a los indicadores de "aval" o de "acreditación"), es solo una pequeña parte de los contenidos de las páginas web que cuentan con una amplia gama de informaciones de otro tipo y que ocupan espacios importantes en dichas páginas: noticias sobre los avances en la estrategia del programa de la OMS sobre la erradicación de la enfermedad en el mundo; información y enlaces a otras asociaciones similares, normativas y legislaciones sobre discapacidad; enlaces a direcciones de profesionales sanitarios e instituciones; un muy importante aspecto de experiencias personales vividas y de formas de afrontar los retos de las secuelas de todo tipo $y$, finalmente, elementos reivindicativos en forma de declaraciones programáticas, manifiestos o similares. Sin embargo, pese a que hay que tener en cuenta estos matices, es evidente que los indicadores de calidad de estas páginas -que en último extremo son un patrón de referencia muy aconsejable, pero no un dogma- mejorarían sustancialmente si se subsanaran algunos de los aspectos contemplados en los indicadores como la autoría, filiación, fechas de creación y actualización, credibilidad, enlaces o contacto, que no son difíciles de cumplimentar.

Finalmente, un tema de mayor calado es que no puede estudiarse este tipo de páginas web solamente desde una perspectiva cuantitativa, sino que es indispensable complementarla con metodología cualitativa que incluya técnicas como entrevistas ad hoc tanto con los promotores como con los usuarios, es decir, conocer quiénes y para qué se han puesto en marcha estas páginas y analizar de forma sistemática la utilidad social y de apoyo que cumplen, de hecho, las mismas. En suma, explorar el papel que desempeña este tipo de comunicación a efectos de lo que son los principios programáticos en los que se basan estos grupos de autoayuda: empoderamiento, toma de decisiones no jerárquica, responsabilidad compartida y una visión holística, no medicalizada en la que el problema físico se integra con lo social, lo económico y lo cultural. 


\section{AGRADECIMIENTOS}

Este trabajo fue realizado dentro del proyecto "Enfermedades emergentes y comunidades de pacientes. El caso de la poliomielitis", financiado por el Ministerio de Ciencia e Innovación, proyectos I+D+I (HAR2009-1468-C03-01). Asimismo forma parte del Proyecto PCI-AECID (D/030704/10), dentro de la convocatoria del Programa de Cooperación Interuniversitaria e Investigación Científica (PCI), perteneciente a las ayudas para Acciones Integradas para el Fortalecimiento Científico e Institucional de la Agencia Española de Cooperación Internacional para el Desarrollo (AECID).

\section{CONFLICTO DE INTERESES}

Los autores declaran que no existe ningún compromiso o vínculo con las entidades financiadoras que pueda ser entendido como un conflicto de intereses.

\section{REFERENCIAS BIBLIOGRÁFICAS}

1. Fundación Vodafone. Tecnologías de la información y comunicaciones y discapacidad: propuestas de futuro [Internet]. Madrid: Fundación Vodafone; 2003 [citado 15 may 2011]. Disponible en: http://tecnologiaedu.us.es/cuestionario/bibliovir/fvodafone.pdf

2. Sanz-Valero J, Castiel LD, Wanden-Berghe C, Juan-Quilis V. Internet y la búsqueda de información en salud pública: desde la relevancia hacia la "revelancia". Gaceta Sanitaria. 2006;20(2):159160.

3. Eysenbach G, Powell J, Kuss Q, Sa ER. Empirical studies assessing the quality of health information for consumers on the World Wide Web: a systematic review. JAMA. 2002;287(20):2691-2700.

4. Guardiola-Wanden-Berghe R, Sanz-Valero J, Wanden-Berghe C. Eating disorders blogs: Testing the quality of information on the Internet. Eating Disorders. 2010;18(2):148-152.

5. Rabeharisoa V, Callon M. La participación de las asociaciones de pacientes en la investigación. Revista Internacional de Ciencias Sociales [Internet]. 2002 [citado 2 may 2011];(171). Disponible: http://www.oei.es/salactsi/volona.pdf

6. Zola IK. Helping one another: a speculative history of self-help movement. Archives of Physical Medicine and Rehabilitation. 1978;60(10):452456.

7. Lock S. Self-groups: the fourth state in medicine? British Medical Journal. 1986;293:15961600.

8. Rabeharisoa V, Callon M. Le pouvoir des malades, L'Association française contre les myopa- thies et la recherche. Paris: Les Presses de I“'École de les Mines; 1999.

9. Dawn O, Braithwaite N, Eckstein J. How people with disabilities communicatively manage assistance: helping as instrumental social support. Journal of Applied Communication Research. 2003;31(1):1-26.

10. Weinberg N, Schmale JD, Uken J, Wessel K. Computer-mediated support groups. Social Work with Groups. 1995; 17(4):43-54.

11. Finn J. An exploration of helping processes in a on-line self-help group focusing on issues of disability. Health and Social Work 1999;24(3):220-231.

12. Latin American and Caribbean Center on Health Sciences Information. Health Information Locator Methodology: Criteria for the Selection of Health Information Sources Available on the Internet [Internet]. $3^{\text {a }}$ Edición. Sao Paulo: BIREME/PAHO/WHO; 2005 [citado 12 may 2011]. Disponible en: http://bvsmodelo.bvsalud.org/ download/lis/LIS-2-CriteriosSelecaoFontes-en.pdf

13. Dublin Core Metadata Initiative. Using Dublin Core [Internet]. Singapore: DCMI; 2005 [citado 30 abr 2011]. Disponible en: http://dublincore.org/ documents/usageguide/

14. Health On the Net Foundation. The HON Code of Conduct for medical and health Web sites [Internet]. Ginebra: HON Foundation; 2009 [citado 5 may 2011]. Disponible en: http://www.hon.ch/HONcode/Conduct.html

15. Web Médica Acreditada. Código de Conducta [Internet]. Barcelona: Colegio Oficial de Médicos de Barcelona; 2002 [citado 5 may 2011]. Disponible en: http://wma.comb.es/esp/codi.htm 
16. Centrale Santé. Net Scoring: criteria to assess the quality of Health Internet information [Internet]. Paris: Centrale Santé; 2001 [citado 6 may 2011]. Disponible en: http://www.churouen.fr/netscoring/netscoringeng.html

17. Martín Martínez B. Valoración de la calidad de las páginas web en gastroenterología, hepatología y nutrición infantil [tesis doctoral]. Barcelona: Universidad de Barcelona, Facultad de Medicina; 2007.

18. Dellavalle RP, Hester EJ, Heilig LF, Drake AL, Kuntzman JW, Graber M, et al. Information science. Going, going, gone: lost Internet references. Science. 2003;302(5646):787-788.

\section{FORMA DE CITAR}

Ballester R, Bueno E, Sanz-Valero J. Información, autoayuda y creación de identidades: las tecnologías de la información y la comunicación (TIC) y las asociaciones de discapacitados físicos. El ejemplo de la poliomielitis. Salud Colectiva 2011;7(Supl 1):S39-S47.

Recibido el 1 de julio de 2011

Aprobado el 16 de septiembre de 2011 